\section{A breath of fresh air}

At this year's BDIA Showcase in

London, Philips announced a range of new products that empower consumers to stay healthy, live well and enjoy life. Philips believes advancements in smart technology and sensors will create meaningful data and be key to helping consumers look after their long-term health.

Philips' HealthSuite platform, a cloud-enabled connected health ecosystem of devices, apps and digital tools, works seamlessly together to empower personalised health and continuous care. HealthSuite is purpose-built to benefit consumers, patients and populations across the health continuum, from healthy living and prevention to diagnosis, treatment and home care.

As well as the Philips Sonicare FlexCare Platinum Connected the company previewed a new connected innovation which will not be officially launched until 2017.

The Philips Sonicare BreathCare is an oral health system which allows consumers to measure, track, and improve breath quality via the Philips Sonicare app using Bluetooth technology. Worldwide, 75\% of consumers worry about bad breath. Philips knows that bad breath is more than just an embarrassing personal issue; it is a proxy for the cleanliness and health of the mouth.

Philips Sonicare BreathCare works with a portable breath analyser device designed to measure bacteria by-products in the mouth with professional-grade accuracy. Supported by Breathometer technology, a custom sensor array analyses a sample of air from the user's mouth in under 30 seconds, and syncs with the Philips Sonicare app via Bluetooth to provide feedback. Using the data captured, the app displays real time results, tracks breath quality patterns, and provides personalised coaching and education to improve the user's breath score, oral care habits, and overall oral health.

Included in the system is Philips Sonicare TongueCare+, a breakthrough tongue cleaning duo powered by Sonicare technology. Philips knows that up to $80 \%$ of bad breath comes from odour-producing bacteria that accumulate and hide deep within the porous surface of the tongue. The Philips Sonicare TongueCare+ combines a specially designed tongue brush head and BreathRx antibacterial tongue spray, which work together to transform a Philips Sonicare power toothbrush into an advanced fresh breath solution.

The innovative tongue brush head, featuring 240 flexible MicroBristles, contours to the tongue to clean away bacteria that a toothbrush and mouthwash cannot reach. The unique combination of ingredients in the Philips Sonicare BreathRx tongue spray is clinically proven to kill the germs that cause bad breath and to neutralise lingering odours. The thick formula coats the tongue and keeps it lubricated for a gentle cleaning experience, while the cool mint flavour leaves the mouth feeling fresh and clean. The Philips Sonicare TongueCare+ is clinically proven to provide all day fresh breath in just 60 seconds.

Philips Sonicare BreathCare will be available for dental professionals from January 2017, six months ahead of its retail launch.

For more information visit the website at www.philips-tsp.co.uk or call 08000567222

\section{BDIA Dental Showcase changes hands}

The British Dental Industry Association (BDIA) has announced that the rights to BDIA Dental Showcase have been sold to George Warman Publications, part of the Mark Allen Group, and a BDIA member, who will now be running the exhibition.

George Warman Publications is the dental communications arm of the Mark Allen Group and publishes magazines and journals for dental professionals including Dental Update, The Dentist, Dental Nursing, Orthodontic Update and associated websites.

Showcase will continue to be known as 'BDIA Dental Showcase' for at least five years and the BDIA will be working very closely with the Mark Allen Group to provide the best possible event for exhibitors and visitors in the future.

\section{Power to the people}

We are all familiar with studies that are evidence-based, but how relevant are they to everyday life? Frequently we find ourselves saying, 'that's all very well in a research laboratory but how does it work in the real world?'

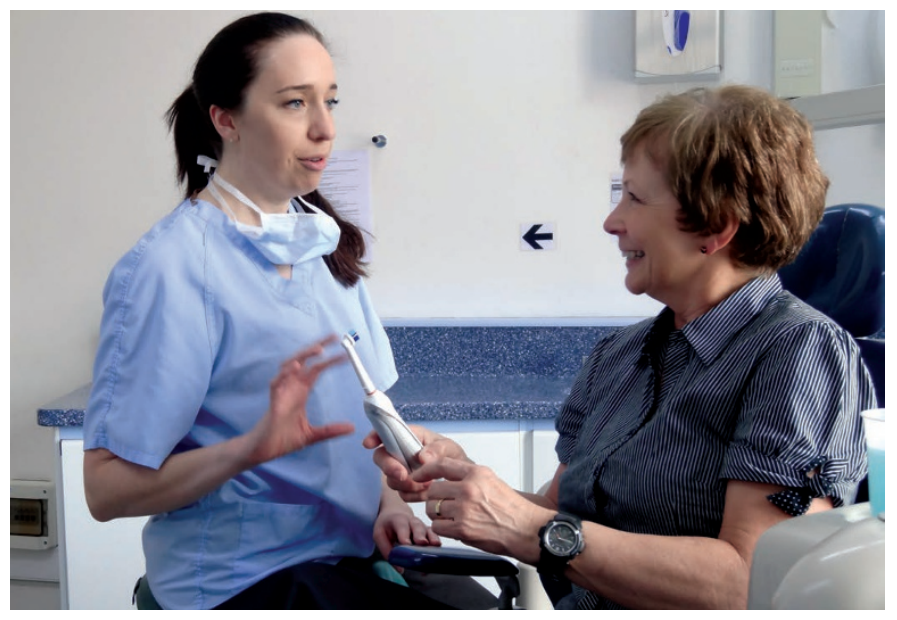

Oral-B wants dental professionals to see the long-term benefits of their products at first hand. Dental professionals are invited to participate in a Patient Evaluation Programme for their new Genius power toothbrush. The patient's oral health status is assessed and recorded at the start of the programme and then again six months later. For those who've never experienced the benefits of an Oral-B power toothbrush, the results can be quite overwhelming. For practitioners it's rewarding to see how technological can be used to change and improve peoples oral care patterns.

Oral-B research has revealed that up to $80 \%$ of people spend insufficient time brushing in at least one zone in their mouth and $60 \%$ of people either didn't brush their back molars at all, or spent insufficient time brushing them. Now, when paired with the Oral-B App 4.1, the Position-Detection Technology in Oral-B Genius tracks brush position, and shows the user how to brush all zones in the mouth equally and evenly. If you would like to participate in this trial programme please contact your Oral-B representative. Your only obligation is to return the survey after six months usage. 